\title{
THE MINIMAL DEGREE OF A FINITE INVERSE SEMIGROUP
}

\author{
BORIS M. SCHEIN
}

\begin{abstract}
The minimal degree of an inverse semigroup $S$ is the minimal cardinality of a set $A$ such that $S$ is isomorphic to an inverse semigroup of one-to-one partial transformations of $A$. The main result is a formula that expresses the minimal degree of a finite inverse semigroup $S$ in terms of certain subgroups and the ordered structure of $S$. In fact, a representation of $S$ by one-to-one partial transformations of the smallest possible set $A$ is explicitly constructed in the proof of the formula. All known and some new results on the minimal degree follow as easy corollaries.
\end{abstract}

If $\rho$ is an isomorphic or a homomorphic representation of an inverse semigroup $S$ by one-to-one partial transformations of a set $A$, then the cardinality of $A$ is denoted by $\delta(\rho)$ and called the degree of $\rho$. Every inverse semigroup has a faithful (that is, isomorphic) representation by one-to-one partial transformations of a set. The minimal degree of a faithful representation of $S$ is called the minimal degree of $S$ and denoted by $\delta(S)$. If the generalized continuum hypothesis is assumed and $S$ is infinite, then $\delta(S)$ is either $|S|$ or the predecessor of $|S|$. This follows from the fact that $\mathscr{J}_{A}$, the symmetric inverse semigroup of all one-to-one partial transformations of an infinite set $A$, has cardinality $2^{|A|}$. In particular, if $|S|$ is a limit cardinal, the $\delta(S)=|S|$. While finding $\delta(S)$ for infinite $S$ is not devoid of interest, we consider only finite inverse semigroups in this paper. Our main result is an exact formula for $\delta(S)$ "modulo groups." Solving semigroup problems "modulo groups" (a semigroup problem reduced to a group problem is considered solved) may raise objections, but in our case a "modulo groups" solution may be the best one can expect. Indeed, if $G$ is a group, then $\delta(G)$, as is easily seen (Lemma 1), is the minimal degree of faithful representations of $G$ by permutations. The minimal degree of groups has been considered for more than a century, no definitive formula for $\delta(G)$ has been found yet, and one can hardly expect that such a formula exists.

If $H$ is a subgroup of $G$, we define $\delta_{G}(H)$, the minimal degree of $H$ in $G$, as the minimal cardinality of a nonempty set $A$ such that there exists a (homomorphic) representation $P$ of $G$ by permutations of $A$ which induces a faithful representation of $H$. Such a representation $\rho$ is called a minimal representation of $H$ in $G$. It seems that this concept has not been considered in the theory of groups, although it belongs to this theory. Observe that $A$ above

\footnotetext{
Received by the editors May 9, 1990 and, in revised form, August 1, 1990.

1980 Mathematics Subject Classification (1985 Revision). Primary 20M30; Secondary 20M18, $20 \mathrm{M} 20$. 
is not empty for the following reason. A trivial group is isomorphic to the group of all permutations of an empty set, and hence, from our point point of view, its degree is 0 (and not 1 !). Yet we want $\mu(D)$, as defined below, to be positive.

One may consider representations of an inverse semigroup $S$ by partial transformations that are not necessarily one-to-one. As proved in [9], the minimal degree of $S$ with respect to such representations coincides with $\delta(S)$.

To state our main result we introduce a few known and less known concepts.

Every inverse semigroup $S$ is naturally (or canonically) ordered by a partial order relation $\omega$, where $s \omega t$ means $s=s s^{-1} t$ for any $s, t \in S$. Here, as usual, $s^{-1}$ is the inverse of $s$, that is, the only element of $S$ such that $s s^{-1} s=s$ and $s^{-1}=s^{-1} s s^{-1}$. Since an inverse semigroup is an ordered set, all usual concepts of the theory of ordered sets can be applied. In particular, an element of $S$ may be the least upper bound (the l.u.b.) of a subset of $S$. An element $s \in S$ is join-irreducible if it is not be the l.u.b. of any subset of $S$ that does not contain $S$. For example, the zero element of $S$, if it exists, is the l.u.b. of the empty subset of elements of $S$, and hence the zero element is not joint-irreducible. If $s<t$ (that is, $s \omega t$ and $s \neq t$ ), then $s$ is called a strict minorant of $t$.

Green's relations $\mathscr{D}$ and $\mathscr{I}$ coincide on every finite semigroup. Thus elements $s$ and $t$ of an inverse semigroup $S$ are $\mathscr{D}$-equivalent if and only if they generate the same principal ideal of $S$ or, equivalently, if $s s^{-1}=u u^{-1}$ and $u^{-1} u=t^{-1} t$ for some $u \in S$. Each $\mathscr{D}$-class of a finite inverse semigroup $S$ is a Brandt groupoid with respect to the partial multiplication induced on it. Each Brandt groupoid is isomorphic to a groupoid of the form $I \times G \times I$, where $I$ is a nonempty set and $G$ is a group. The product of its elements $(i, g, j)$ and $(k, h, l)$ is defined precisely when $j=k$, in which case it is equal to $(i, g h, l)$. Thus every $\mathscr{D}$-class $D_{s}$ of $S$ is isomorphic to a Brandt groupoid of the form $I \times G \times I$. Here $G$ is isomorphic to any of the maximal subgroups of $D_{s}$, for example, $G \simeq H_{s s^{-1}}$, where $H_{s s^{-1}}$ is the $\mathscr{H}$-class of $S$ that contains $s s^{-1}$ (that is, $H_{s s^{-1}}$ is the maximal subgroup of $S$ whose identity element is $s s^{-1}$ ). The cardinality of the set $I$ equals the cardinality of the set of all idempotents of $D_{s}$ because idempotents of $I \times G \times I$ are precisely the triples of the form $(i, e, i)$, where $e$ is the identity element of $G$. Let $l(D)$ denote the cardinality of the set of all idempotents in a $\mathscr{D}$-class $D$.

A $\mathscr{D}$-class $D$ is called join-irreducible if it contains a join-irreducible element. It is proved in Lemma 2 that all elements of a join-irreducible $\mathscr{D}$-class are join-irreducible. In the sequel $\mathbf{D}$ denotes the set of all join-irreducible $\mathscr{D}$-classes of $S$.

Let $H_{e}$ be the maximal subgroup of $S$ with $e$ as its identity element. Define the following subset $S_{e}$ of $H_{e}: h \in S_{e}$ if and only if $f \leq h$ for all $f$ in $S$ such that $f<e$. Thus $h \in S_{e}$ if $h h^{-1}=h^{-1} h=e$ and $e$ and $h$ have precisely the same strict minorants in $S$. For example, if $h \in S_{e}$ and $h \neq e$, then $e$ and $h$ have the same strict minorants, and hence $e$ is not the l.u.b. of the set of its strict minorants. Thus, if $S_{e} \neq\{e\}$, then $e$ is join-irreducible. It is proved in Lemma 3 that $S_{e}$ is a normal subgroup of $H_{e}$.

Let $e \in D$, where $D$ is a join-irreducible $\mathscr{D}$-class of $S$. Define $\mu(D)=$ $\delta_{H_{e}}\left(S_{e}\right)$. We prove in Lemma 4 that $\mu(D)$ is well defined, that is, it does not depend on the choice of $e$ in $D$. It follows from our definition of $\delta_{G}(H)$ that $\mu(D) \geq 1$. Obviously, $\mu(D)=1$ if and only if $S_{e}$ is a trivial group. 
Now we are ready to state our main result.

Theorem. If $S$ is a finite inverse semigroup, then

$$
\delta(S)=\sum\{l(D) \cdot \mu(D): D \in \mathbf{D}\} .
$$

Proof. Our equality follows from Lemmas 6 and 7.

Lemma 1. If $G$ is a group, then $\delta(G)$ is the minimal degree of faithful representations of $G$ by permutations.

Proof. Let $R$ be a representation of $G$ by one-to-one partial transformations of a set $A$. Then $R(e)$ is the identity mapping of a subset $B$ of $A$. For every $g \in G, g g^{-1}=e$ and $e g=g$. It follows that $R(g) R\left(g^{-1}\right)=R(e)$ and $R(e) R(g)=R(g)$. Therefore, the domains of $R(g)$ and $R(e)$ coincide, that is, $B$ is the domain of $R(g)$. Analogously, $B$ is the range of $R(g)$. Thus $R(g)$ is a permutation of $B$. Restricting $R(g)$ to $B$ for all $g \in G$ we obtain a representation $R_{B}$ by permutations of $B$. If $R$ is faithful, so is $R_{B}$, and hence if $R$ is minimal (that is, it is faithful and $\delta(G)=|A|$ ), then $B=A$ and $R$ is a representation of $G$ by permutations of a set.

Lemma 2. All elements of a join-irreducible $\mathscr{D}$-class of an inverse semigroup are join-irreducible.

Proof. Let $s, t \in D$. There are $u, v \in D$ such that $s=u t v$ and $t=u^{-1} s v^{-1}$. If $t$ is the l.u.b. of elements $t_{i}, 1 \leq i \leq n$, then $t_{i} \leq t$, and so $s_{i}=u t_{i} v \leq$ $u t v=s$ for all $i$. Let $s_{i} \leq w$ for all $i$. Then $t_{i}=u^{-1} u t_{i} v v^{-1} \leq u^{-1} s_{i} v^{-1} \leq$ $u^{-1} w v^{-1}$ for all $i$, so that $t \leq u^{-1} w v^{-1}$. Now $s=u t v \leq u u^{-1} w v^{-1} v \leq w$. Thus $s$ is the l.u.b. of elements $s_{i}, 1 \leq i \leq n$. If $s$ is join-irreducible, $s=s_{i}$ for some $i$. It follows that $t=u^{-1} s v^{-1}=u^{-1} s_{i} v^{-1}=t_{i}$, and hence $t$ is join-irreducible.

Lemma 3. $S_{e}$ is a normal subgroup of $H_{e}$.

Proof. Let $u, v \in S_{e}$. If $f<e$, then $f<u$ and $f<v$. Since $f$ is idempotent, $f<v^{-1}$, and hence $f=f^{2} \leq u v^{-1}$, where $u v^{-1} \in H_{e}$. Thus $f \neq u v^{-1}$, so that $f<u v^{-1}$. It follows that $u v^{-1} \in S_{e}$. Also, $e \in S_{e}$, so that $S_{e} \neq \varnothing$. Therefore, $S_{e}$ is a subgroup of $H_{e}$.

If $u \in S_{e}$ and $v \in H_{e}$, then $f<u$ for every $f$ such that $f<e$. It follows that $v f v^{-1} \leq v e v^{-1}=e$. If $v f v^{-1}=e$, then $f=e f e=v^{-1} v f v^{-1} v=$ $v^{-1} e v=e$, and hence $v f v^{-1}<e$. Therefore, $v f v^{-1}<u$, so that $f=$ $v^{-1} v f v^{-1} v<v^{-1} u v$. Thus $v^{-1} u v \in S_{e}$ and $S_{e}$ is a normal subgroup of $H_{e}$.

Lemma 4. $\mu(D)$ does not depend on the choice of $e$ in $D$.

Proof. Let $e$ and $f$ be idempotents in a join-irreducible $\mathscr{D}$-class $D$. Then the groups $H_{e}$ and $H_{f}$ are isomorphic, for there exists $v \in D$ such that the mapping $i^{v}(x)=v^{-1} x v$ for all $x \in H_{e}$ is an isomorphism of $H_{e}$ onto $H_{f}$. To see that $i^{v}\left(S_{e}\right)=S_{f}$, assume that $u \in S_{e}$. If $k<f$, then $v k v^{-1}<v f v^{-1}=e$, so that $v k v^{-1}<u$. Thus $k=f k f=v^{-1} v k v^{-1} v<v^{-1} u v=i^{v}(u)$ and $i^{v}(u) \in S_{f}$, i.e., $i^{v}$ maps $S_{e}$ into $S_{f}$. The inverse isomorphism of $S_{f}$ onto $S_{e}$ maps $S_{f}$ into $S_{e}$, so that $i^{v}$ maps $S_{e}$ onto $S_{f}$, and hence groups $S_{e}$ and $S_{f}$ are isomorphic. 
The reader is supposed to be familiar with the theory of representations of inverse semigroups by one-to-one partial transformations of sets as developed in [6]. Those readers who are not as fluent in Russian as they undoubtedly wish they were, can find an exposition of this theory in English in Chapter 7 of [1], Chapter V of [4], or Chapter IV of [5]. To make this paper reasonably self-contained, we give a very brief description of some concepts of this theory here.

A subset $T$ of an inverse semigroup $S$ is called majorantly closed if $s \in T$ and $s \leq t$ imply $t \in T$ for all $s, t \in S$. If $U$ is a subset of $S$, then $\bar{U}$ denotes its majorant closure, that is, $t \in \bar{U}$ if and only if $s \leq t$ for some $s \in U$. If $F$ is an inverse subsemigroup of $S$, its majorant closure $\bar{F}$ is a majorantly closed inverse subsemigroup of $S$.

Let $T$ be a majorantly closed inverse subsemigroup of $S, s \in S$ and $s s^{-1} \in$ $T$. The subset $\overline{T s}$ of $S$ is called a right coset of $T$. If $s s^{-1} \notin T$, the right coset of $T$ is not defined. The set of all right cosets of $T$ is denoted by $S / T$. If $s^{-1} s \in T$, then $\overline{s T}$. is called a left coset of $T$. Right (left) cosets of $T$ either are disjoint or coincide, and they need not cover $S$. The number of right cosets of $T$ is called the index of $T$ in $S$ And denoted by $[S: T]$. Thus $[S: T]=|S / T|$. If $\overline{T S}$ is a right coset of $T$, then $\overline{S^{-1} T}$ is a left coset. This gives a one-to-one correspondence between right and left cosets of $T$, so that $[S: T]$ is the number of left cosets of $T$.

Let $T$ be a majorantly closed inverse subsemigroup of an inverse semigroup $S$. The transitive representation of $S$ by one-to-one partial transformations of the set $S / T$ is denoted by $P_{T}$. For every $s \in S$ and every right coset $\overline{T t}$ of $T, \overline{T t}$ belongs to the domain of $P_{T}(s)$ precisely if the right coset $\overline{T t s}$ exists (that is, if $\left.(t s)(t s)^{-1} \in T\right)$, in which case the image of $\overline{T t}$ under $P_{T}(s)$ is $\overline{T t s}$. Thus $\delta\left(P_{T}\right)=[S: T]$. Also $P_{T}(s)=P_{T}(t)$ for $s, t \in S$ if and only if $(\forall x, y \in S)\left[x s y \in T_{i} \Leftrightarrow x t y \in T_{i}\right]$.

If $S$ is finite, every majorantly closed inverse subsemigroup $T$ of $S$ is a majorant closure of a subgroup of $S$. Indeed, if $e$ is the least idempotent of $T$ and $F$ the maximal subgroup of $T$ that contains $e$, then $\bar{F} \subset T$. If $h \in T$, then $e h e \in T$. It follows from $e(e h e)=(e h e) e=e h e$ that $e h e \in F$. The inequality $e h e \leq h$ shows that $h \in \bar{F}$. Thus $T=\bar{F}$.

Lemma 5. Let $T=\bar{F}$ be a majorantly closed inverse subsemigroup of an inverse semigroup $S$, where $F$ is a maximal subgroup of $T$. Let $G$ be the maximal subgroup of $S$ that contains $F$, and $D$ the $\mathscr{D}$-class of $S$ that contains $G$. Then $\delta\left(P_{T}\right)=[G: F] \cdot l(D)$.

Proof. Clearly, $\delta\left(P_{T}\right)=[S: T]$. Let $\overline{T s} \in S / T$. Then $F s \subset T s$, and if $t s \in T s$ for $t \in T$, then $g \leq t$ for some $g \in F$ and $g s \leq t s$. Thus $\overline{T s}=\overline{F s}$. Since $s s^{-1} \in T$, we see that $(e s)(e s)^{-1}=e s s^{-1} e=e$, and hence $e s \in D$ and $F e s=(F e) s=F s$. Therefore, all right cosets of $T$ have the form $\overline{T u}=\overline{F u}$ with $u \in D$. If $\overline{T u}=\overline{T v}$ for $u, v \in D$, then $u v^{-1} \in T$, so that $u u^{-1}$, $v v^{-1} \in T$ and $u u^{-1}=v v^{-1}=e$. If $g \leq u v^{-1}$ for $g \in F$, then $g v \leq$ $u v v^{-1} \leq u$. Now,

$$
(g v)(g v)^{-1}=g v v^{-1} g^{-1}=g e g^{-1}=e=u u^{-1},
$$

so that $g v=u$ or, equivalently, $\overline{F u}=\overline{F v}$. Conversely,

$$
\overline{F u}=\overline{F v} \Rightarrow u v^{-1} \in F \Rightarrow u v^{-1} \in T \Rightarrow \overline{T u}=\overline{T v} \text {. }
$$


Thus $\overline{T u}=\overline{T v}$ if and only if $\overline{F u}=\overline{F v}$. It follows that the number of right cosets of $T$ in $S$ equals the number of right cosets of $F$ in $D$.

Since $D$ is finite, it is a Brandt groupoid under the partial multiplication induced by that of $S$. Therefore, $D$ is isomorphic to a Brandt groupoid of the form $I \times K \times I$, where $I$ is a nonempty set, $K$ is a group, the product of triples $(i, g, j)$ and $(k, h, l)$ is defined precisely when $j=k$, and $(i, g, j)(j, h, l)=(i, g h, l)$. The group $K$ is isomorphic to any maximal subgroup of the groupoid, and so we assume that $K=G$. Idempotents of $I \times K \times I$ have the form $(i, e, i)$, where $e$ is the identity element of $K$, and hence there are precisely as many idempotents in $D$ as there are elements in $I$, so that $l(D)=|I|$. Every subgroup of $I \times G \times I$ is of the form $M=\{i\} \times F \times\{i\}$, where $F$ is a subgroup of $G$ and $i \in I$. If $u \in I \times G \times I$, then $u u^{-1} \in M$ means that $u=(i, g, j)$ for some $g \in G$ and $j \in I$. Also, $M u \neq \varnothing$ if and only if $u=(i, g, j)$ for some $g \in G$ and $j \in I$, so that $M u=\{i\} \times F g \times\{j\}$. Thus, if $u, v \in I \times G \times I$ and both $M u$ and $M v$ are not empty, then $M u=M v$ exactly if $u=(i, g, j)$ and $v=(i, h, j)$ for some $g, h \in G$ and $j \in I$, in which case $F g=F h$. Therefore, the number of different nonempty sets $M u$ is the number of different right cosets of $F$ in $G$ multiplied by the number of all elements $j$ in $I$. In other words, this number is the index of $F$ in $G$ multiplied by the cardinality of $I$, that is, $[G: F] \cdot l(D)$. The number of $M u$ in $I \times G \times I$ equals the number of $F u$ in $D$, which, in its own turn, equals the number of right cosets of $T$ in $S$.

Lemma 6. $\delta(S) \leq \sum\{l(D) \cdot \mu(D): D \in \mathbf{D}\}$.

Proof. We will construct a representation $P$ of $S$. Choose a collection of subgroups of $S$ in the following way. From each $D \in \mathbf{D}$ choose an idempotent $e \in D$ and consider the group $S_{e}$. Let $R_{e}$ be a minimal representation of $S_{e}$ in $H_{e}$ by permutations. Then $R_{e}$ is a sum of $n_{e}$ transitive representations of $H_{e}$, and each of these representations is similar to a representation $R_{i}$ by permutations of right cosets of a subgroup $F_{i}$ of $H_{e}, i=1,2, \ldots, n_{e}$. The degree $\delta\left(R_{e}\right)$ of $R_{e}$ is the sum of degrees of these representations $R_{i}$, and thus it is the sum of indexes of $F_{i}$ in $H_{e}$. Let $T_{i}=\bar{F}_{i}$. The transitive representation $P_{T_{i}}$ of $S$ by one-to-one partial transformations of the set $S / T_{i}$ of right cosets of $T_{i}$ will be denoted by $P_{i}$. By Lemma $5, \delta\left(P_{i}\right)=\imath(D) \cdot\left[S: T_{i}\right]$. Let $P_{e}$ be the sum of representations $P_{i}, 1 \leq i \leq n_{e}$. Then

$$
\delta\left(P_{c}\right)=\sum\left\{\delta\left(P_{i}\right): 1 \leq i \leq n_{e}\right\}=\Sigma\left\{l(D) \cdot\left[S: T_{i}\right]: 1 \leq i \leq n_{e}\right\}=l(D) \cdot \mu(D) .
$$

Let $P$ be the sum of $P_{e}$ for all $D \in \mathbf{D}$. Then $\delta(P)=\sum\{l(D) \cdot \mu(D)$ : $D \in \mathbf{D}\}$. If $P$ is faithful, then $\delta(S) \leq \delta(P)$. Thus it suffices to prove that $P$ is faithful. We do that in a few steps. It suffices to prove the implication $P(s)=P(t) \Rightarrow s \leq t$ for $s, t \in S$. Indeed, if it holds and $P(s)=P(t)$, then $s \leq t$ and, interchanging $s$ and $t$, we obtain $t \leq s$, so that $s=t$ and $P$ is faithful.

Therefore, assume that $P(s)=P(t)$. If $z \leq s$ for no join-irreducible elements $z \in S$, then $s$ is the join of an empty set of join-irreducible elements, i.e. $s=0$, so that $s \leq t$. Let $z \leq s$ for some join-irreducible element $z \in S$. Our goal is to prove that $z \leq t$. Since $z \leq s$, we obtain $z=z z^{-1} s$, and hence

$$
P(z)=P\left(z z^{-1} s\right)=P\left(z z^{-1}\right) P(s)=P\left(z z^{-1}\right) P(t)=P\left(z z^{-1} t\right) .
$$


Let $D$ be the $\mathscr{D}$-class of $S$ that contains $z$. Obviously, $D \in \mathbf{D}$. Let $e$ be the idempotent of $D$ chosen in our construction of $P$. Clearly, $P_{e}(z)=P_{e}\left(z z^{-1} t\right)$. Since $P_{e}$ is the sum of representations $P_{i}, i=1, \ldots, n_{e}$, we obtain $P_{i}(z)=$ $P_{i}\left(z z^{-1} t\right)$ for all $i$. This means that $(\forall x, y \in S)\left[x z y \in T_{i} \Leftrightarrow x z z^{-1} t y \in T_{i}\right]$.

Let $v^{-1} v=e$ and $v v^{-1}=z^{-1} z$, so that $v \in D$. If $x=(z v)^{-1}$ and $y=v$, then

$$
(z v)^{-1} z v \in T_{i} \Leftrightarrow(z v)^{-1} z z^{-1} t v \in T_{i} .
$$

But $(z v)^{-1} z v=e \in T_{i}$, and hence $v^{-1} z^{-1} t v=(z v)^{-1} z z^{-1} t v \in T_{i}$. Clearly,

$$
v^{-1} z^{-1} t v=e\left(v^{-1} z^{-1} t v\right) e \in e T_{i} e=F_{i},
$$

where $F_{i}$ is a maximal subgroup of $T_{i}$ such that $e \in F_{i}$. A straightforward argument shows that $R_{i}\left(v^{-1} z^{-1} t v\right)=R_{i}(e)$ for all $v$ such that $v^{-1} v=e$ and $v v^{-1}=z^{-1} z$, where $R_{i}$ is a representation of the group $H_{e}$ by permutations of the right cosets of $F_{i}$ in $H_{e}$. This holds for all $i=1, \ldots, n_{e}$, and hence $R_{e}\left(v^{-1} z^{-1} t v\right)=R_{e}(e)$ for all $v$ such that $v^{-1} v=e$ and $v v^{-1}=z^{-1} z$.

To prove that $z \leq t$ we use an inductive procedure over join-irreducible idempotents $e$.

(1) If $e$ is a minimal element in the set of all join-irreducible idempotents, then $S_{e}=H_{e}$. Indeed, the implication $f<e \Rightarrow f<g$ holds for all $g \in H_{e}$ and all join-irreducible idempotents $f$, because $f<e$ is always false. Since $R_{e}$ is a minimal representation of the group $S_{e}$ in $H_{e}$ and $S_{e}=H_{e}$, we see that $R_{e}$ is a minimal representation of $H_{e}$ by permutations, and hence $R_{e}$ is faithful. It follows that $u z v=u z z^{-1} t v$. Therefore, $z=u^{-1} u z v v^{-1}=$ $u^{-1} u z z^{-1} t v v^{-1} \leq t$.

(2) Let $z \leq s \Rightarrow z \leq t$ for all $z \in D_{f}$ and all idempotents $f$ such that $D_{f}<$ $D_{e}$. Here $D_{f}<D_{e}$ means that $D_{f} \neq D_{e}$ and $D_{f} \leq D_{e}$ in the sense of the usual ordering on $\mathscr{D}$-classes of $S$. Let $g$ be a join-irreducible idempotent such that $g<e$. Then $D_{g}<D_{e}$ and, since $v g v^{-1}$ is idempotent, $z\left(v g v^{-1}\right) \leq z \leq s$, so that

$$
\begin{aligned}
{\left[z\left(v g v^{-1}\right)\right]^{-1}\left[z\left(v g v^{-1}\right)\right] } & =\left(v g v^{-1}\right) z^{-1} z\left(v g v^{-1}\right) \\
& =\left(v g v^{-1}\right) v v^{-1}\left(v g v^{-1}\right)=(v g)(v g)^{-1}
\end{aligned}
$$

and $(v g)^{-1}(v g)=g v^{-1} v g=g e g=g$. Therefore, $z(v g v)^{-1} \in D_{g}$, and hence

$$
z\left(v g v^{-1}\right) \leq t \quad \text { and } \quad\left[z\left(v g v^{-1}\right)\right]^{-1} t=\left[z\left(v g v^{-1}\right)\right]^{-1}\left[z\left(v g v^{-1}\right)\right]=v g v^{-1} .
$$

Thus

$$
\begin{aligned}
g v^{-1} z^{-1} t v & =g\left(v^{-1} v\right) v^{-1} z^{-1} t v=\left(v^{-1} v\right) g v^{-1} z^{-1} t v \\
& =v^{-1}\left[z\left(v g v^{-1}\right)\right]^{-1} t v=v^{-1} v g v^{-1} v=e g e=g .
\end{aligned}
$$

It follows that $g \leq v^{-1} z^{-1} t v$. However, $v^{-1} z^{-1} t v \in H_{e}$ and $g \notin H_{e}$, so that $g<v^{-1} z^{-1} t v$.

Thus $g<v^{-1} z^{-1} t v$ for all join-irreducible $g$ such that $g<e$. Let $f$ be an idempotent such that $f<e$. Then $D_{f}<D_{e}$. Obviously, each element of $S$ is a join of join-irreducible elements. Let $f$ be a join of join-irreducible elements $g_{1}, \ldots, g_{k}$. Clearly, $g_{i} \leq f<e$, and hence $g_{i}<e$ and $g_{i}$ is a joinirreducible idempotent for every $i=1, \ldots, k$. It follows that $g_{i}<v^{-1} z^{-1} t v$ 
for all $i$, and hence $f<v^{-1} z^{-1} t v$. Thus $v^{-1} z^{-1} t v \in H_{e}$, and if $f<e$ then $f<v^{-1} z^{-1} t v$. Therefore, $v^{-1} z^{-1} t v \in S_{e}$.

We proved earlier that $R_{e}\left(v^{-1} z^{-1} t v\right)=R_{e}(e)$. Now we see that both $v^{-1} z^{-1} t v$ and $e$ belong to $S_{e}$. Since $R_{e}$ is a minimal representation of $S_{e}$ in $H_{e}$, it is faithful on $S_{e}$. Thus $v^{-1} z^{-1} t v=e$, and hence

$$
z=z z^{-1} z z^{-1} z=z v v^{-1} v v^{-1}=z v e v^{-1}=z v v^{-1} z^{-1} t v v^{-1} \leq t .
$$

It follows that $z \leq t$.

We proved that $z \leq s \Rightarrow z \leq t$ for all join-irreducible $z$ in $S$. If $s$ is the l.u.b. of join-irreducible elements $z_{1}, z_{2}, \ldots, z_{k}$, then $z_{i} \leq s$ for all $i=1,2, \ldots, k$, and hence $z_{i} \leq t$ for all $i$. Therefore, $s \leq t$.

Lemma 7. $\sum\{l(D) \cdot \mu(D): D \in \mathbf{D}\} \leq \delta(S)$.

Proof. Let $R$ be a faithful representation of $S$ by one-to-one partial transformations of a finite set. Then $R$ is a sum of transitive representations and every transitive representation is similar to $P_{T}$, where $T$ is a majorantly closed inverse subsemigroup of $S$ (see [6]). We can assume that $R$ is a sum of a family $\left\{P_{T_{i}}\right\}_{i \in I}$ of transitive representations. Also, assume that $T_{i}=\bar{F}_{i}$, where $F_{i}$ is a subgroup of $T_{i}$. Let $e_{i}$ denote the identity element of $F_{i}$, and let $e_{i}$ belong to a $\mathscr{D}$-class $D_{i}$ of $S$.

Suppose that $D \in \mathbf{D}$ and $e^{2}=e \in D$. Let $s \in S$ be such that $x^{-1} e_{i} x \leq$ $e \Rightarrow x^{-1} e_{i} x \leq s$ for all $i \in I$ and $x \in S$ such that $x^{-1} e_{i} x \in D_{i}$. If $x e y \in T_{i}$ for some $i \in I$, then $x e y \leq x y$ and $(x e y)^{-1}(x e y) \leq y^{-1} e y$, so that $x y$, $y^{-1} e y \in T_{i}$. Also, $y^{-1} e y \in T_{i}$ implies $e_{i} \leq y^{-1} e y \leq y^{-1} y$. It follows that $y e_{i} y^{-1} \in D_{i}$ and $y e_{i} y^{-1} \leq y y^{-1} e y y^{-1} \leq e$. By our assumption, $y e_{i} y^{-1} \leq s$, so that $e_{i}=y^{-1} y e_{i} y^{-1} y \leq y^{-1} s y$ and $y^{-1} s y \in T_{i}$. Clearly, $(x y)\left(y^{-1} s y\right) \leq x s y$, and so $x s y \in T_{i}$. Thus $(\forall i \in I)(\forall x, y \in S)\left[x e y \in T_{i} \Rightarrow x s y \in T_{i}\right]$, which implies $R(e) \subset R(s)$ (see [6]). Since $R$ is faithful, we obtain $e \leq s$. It follows that $e$ is the l.u.b. of elements of the form $x^{-1} e_{i} x$ for all $x \in S$ such that $x^{-1} e_{i} x \in D_{i}$. Since $e$ is join-irreducible, we have $e=x^{-1} e_{i} x \in D_{i}$ for some $i \in I$, and so $D=D_{i}$. Let $I_{D}=\left\{i \in I: D=D_{i}\right\}$.

Suppose that $D_{i}=D_{j}$. Then $e_{i}=u u^{-1}$ and $e_{j}=u^{-1} u$ for some $u \in$ $S$. The representation $P_{T_{i}}$ is similar to the representation corresponding to the majorantly saturated inverse subsemigroup $\overline{u^{-1} T_{i} u}$ (see [6]), and so we can replace $P_{T_{i}}$ by this similar representation. It follows from $T_{i}=\bar{F}_{i}$ that $\overline{u^{-1} T_{i} u}=\overline{u^{-1} F_{i} u}$. But $e_{j}=u^{-1} e_{i} u \in \overline{u^{-1} F_{i} u}$. Thus, without loss of generality, we can assume that $e_{i}=e_{j}$ for all $i, j \in I$ such that $D_{i}=D_{j}$.

Choose $D \in \mathbf{D}$ and consider a sum $R_{D}$ of all representations $P_{T_{i}}, i \in I_{D}$. All idempotents $e_{j} \in D$ considered above are equal, and so we skip the index $j$ and write just $e_{j}=e$. Restricting $R_{D}$ to the subgroup $H_{e}$ of $S$, we obtain a representation $P_{D}$ of $H_{e}$ that is the sum of transitive representations of $H_{e}$ corresponding to all subgroups $F_{i} \subset D$. Let $h \in S_{e}$ and $P_{D}(h)=P_{D}(e)$. Then $R_{D}(h)=R_{D}(e)$ and $P_{F_{i}}(h)=P_{F_{i}}(e)$ for all $i \in I_{D}$, where $P_{F_{i}}$ denotes the representation of $H_{e}$ over the right cosets of its subgroup $F_{i}$. Let xey $\in T_{i}$ for some $i \in I$. Since $x e y \leq x y$ and $(x e y)^{-1}(x e y) \leq y^{-1} e y$, we obtain $x y, y^{-1} e y \in T_{i}$. Thus $e_{i} \leq y^{-1} e y$. As we have seen above, $y e_{i} y^{-1} \leq e$, where $y e_{i} y^{-1} \in D_{i}$. If $y e_{i} y^{-1}<e$, then $y e_{i} y^{-1}<h$, because $h \in S_{e}$. Then $e_{i}=y^{-1} y e_{i} y^{-1} y \leq y^{-1} h y$, and so $y^{-1} h y \in T_{i}$. Since $(x y)\left(y^{-1} h y\right) \leq x h y$, we obtain $x h y \in T_{i}$. Now let $y e_{i} y^{-1}=e$. Then $e \in D_{i}, D_{i}=D$, and $e_{i}=e$. It 
follows from $y e y^{-1}=e$ that

$$
\begin{aligned}
y^{-1} e & =y^{-1} y e y^{-1}=e y^{-1} y y^{-1}=e y^{-1} \in H_{e}, \quad \text { and } \\
e y^{-1} y & =(y e)^{-1} y=(e y)^{-1}(e y)=e,
\end{aligned}
$$

so that $\left(F_{i} e y^{-1}\right) e=\left(F_{i} y^{-1}\right) e=\left(F_{i} e y^{-1}\right)$ for every $i \in I_{D}$. Since $P_{D}(h)=$ $P_{D}(e)$, we obtain $\left(F_{i} e y^{-1}\right) h=\left(F_{i} e y^{-1}\right)$. Thus

$$
F_{i} y^{-1} h y=\left(F_{i} e\right) y^{-1} h y=\left(F_{i} e y^{-1}\right) h y=\left(F_{i} e y^{-1}\right) y=F_{i}\left(e y^{-1} y\right)=F_{i} e=F_{i}
$$

and $y^{-1} h y \in F_{i} \subset T_{i}$. Therefore, $x h y \in T_{i}$.

We proved that $(\forall i \in I)(\forall x, y \in S)\left[\left(x e y \in T_{i} \Rightarrow x h y \in T_{i}\right]\right.$. Therefore (see [6]), $R(e) \subset R(h)$. Since $R$ is faithful, we obtain $e \leq h$, so that $e=e h=h$. Thus $P_{D}$ is faithful on $S_{e}$. Then

$$
\mu(D)=\delta_{H_{e}}\left(S_{e}\right) \leq \delta\left(P_{D}\right)=\sum\left\{\left[H_{e}: F_{i}\right]: i \in I_{D}\right\} .
$$

By Lemma 5,

$$
l(D) \cdot \mu(D) \leq \sum\left\{l(D) \cdot\left[H_{e}: F_{i}\right]: i \in I_{D}\right\}=\delta\left(R_{D}\right),
$$

and hence

$$
\sum\{l(D) \cdot \mu(D): D \in \mathbf{D}\} \leq \sum\left\{\delta\left(R_{D}\right): D \in \mathbf{D}\right\} \leq \delta(R)
$$

for every faithful representation $R$ of $S$. Thus $\sum\{l(D) \cdot \mu(D): D \in \mathbf{D}\} \leq$ $\delta(S)$.

Using the Theorem, we may easily obtain all known results on $\delta(S)$. Consider the following example. A (finite) inverse semigroup is called unruly if $\delta(S)=|S|$. These inverse semigroups were described in [7]. We obtain this result as Corollary 1. First we need a few known definitions. A semilattice of groups is an inverse semigroup $S$ that is a union of its maximal subgroups $H_{e}$, $e \in E$, where $E$ is the semilattice of idempotents of $S$. If $e, f \in E$ and $f<e$, then the mapping $\varphi_{e, f}: H_{e} \rightarrow H_{f}$ defined by $h \varphi_{e, f}=h f$ for every $h \in H_{e}$ is called a structural homomorphism of $S$. This structural homomorphism is called trivial if its range coincides with $\{f\}$, it is called injective if it is one-to-one. An idempotent $e$ is called isolated if $H_{e}=\{e\}$.

Corollary 1 [7]. A finite inverse semigroup $S$ is unruly (that is, $\delta(S)=|S|$ ), if and only if $S$ satisfies the following conditions:

(1) $S$ is a semilattice of groups of the following types: (i) cyclic groups of prime power order; (ii) generalized quaternion groups; (iii) Klein's four-group; (iv) trivial group;

(2) no structural homomorphisms are injective except those defined on trivial subgroups; all structural homomorphisms defined on Klein's four-groups are trivial;

(3) $S$ has no zero, and no isolated idempotent is a join of two of its strict minorants. ${ }^{1}$

Proof. By our Theorem, $S$ is unruly if and only if $\sum\{l(D) \cdot \mu(D): D \in \mathbf{D}\}=$ $|S|$. Clearly, $\mu(D) \leq\left|H_{e}\right|$, where $e \in D$. Thus $l(D) \cdot \mu(D) \leq l(D)^{2} \cdot\left|H_{e}\right|=|D|$, so that $S$ is unruly if and only if $|D|=l(D) \cdot \mu(D)$ and every $\mathscr{D}$-class $D$ of

\footnotetext{
${ }^{1}$ This property corrects an inaccuracy in the statement of this result in [7].
} 
$S$ is join-irreducible, which, by Lemma 2, means that all idempotents of $S$ are join-irreducible. The first condition means that $l(D)^{2} \cdot\left|H_{e}\right|=l(D) \cdot \mu(D)$, that is, $l(D) \cdot\left|H_{e}\right|=\mu(D) \leq\left|H_{e}\right|$. Equivalently, $\mu(D)=\left|H_{e}\right|$, and $l(D)=1$, so that $D$ contains a single idempotent. Therefore, $D=H_{e}$ and $S$ is a semilattice of groups $H_{e}$. Obviously, $\mu\left(D_{e}\right) \leq \delta\left(H_{e}\right)$ for $H_{e} \neq\{e\}$, and thus all nontrivial groups $H_{e}$ are unruly. Amazingly, unruly groups do not seem to be described in the group literature. Their description was given in [7], and they are precisely the groups of types (i), (ii), and (iii). If $e, f \in E, f<e$, and $h \in S_{e}$, then $f<h$, and hence $e \varphi_{e, f}=e f=f=h f=h \varphi_{e, f}$. If $\varphi_{e, f}$ is injective, then $e=h$, and hence $S_{e}=\{e\}$. Then $\left|H_{e}\right|=\mu\left(H_{e}\right)=1$, and hence $H_{e}$ is trivial. Now suppose that $H_{e}$ is a Klein four-group. As we have just seen, $S_{e} \neq\{e\}$. For $\left|S_{e}\right|=2$ an easy computation shows that $\mu\left(H_{e}\right)=2 \neq \delta\left(H_{e}\right)$. Therefore, $\left|S_{e}\right|=4$, and hence $S_{e}=H_{e}$. This means that $\mu\left(H_{e}\right)=\left|H_{e}\right|$ and all structural homomorphisms $\varphi_{e, f}$ for $f<e$ are trivial. As the zero element is not joinirreducible, $S$ has no zero. Therefore, if $S$ is unruly, it satisfies conditions (1)-(3) of our corollary.

Conversely, if $S$ satisfies (1)-(3), we need to prove that $\mu\left(H_{e}\right)=\left|H_{e}\right|$ for every maximal subgroup $H_{e}$ and that all idempotents of $S$ are join-irreducible. Indeed, $\mu\left(H_{e}\right)=\left|H_{e}\right|$ for trivial groups $H_{e}$. If $H_{e}$ belongs to one of the types (i) or (ii), then it contains the least nontrivial subgroup. This subgroup is normal, and hence it is contained in the kernel of every noninjective homomorphism of $H_{e}$. Since all structural homomorphisms of $H_{e}$ are not injective, this least subgroup is contained in all of them, and so it is contained in $S_{e}$. This shows that $S_{e}$ is not trivial, and hence $e$ is join-irreducible. Also, it is easy to check that $\mu\left(H_{e}\right)=\left|H_{e}\right|$ in this case. If $e$ is the least idempotent of $S$, then $S_{e}=H_{e} \neq\{e\}$, and hence $e$ is join-irreducible and $\mu\left(H_{e}\right)=\left|H_{e}\right|$. Now, if $H_{e}$ is Klein's four-group, then all structural homomorphisms of $H_{e}$ are trivial, and hence $H_{e}=S_{e}$, and again $e$ is join-irreducible and $\mu\left(H_{e}\right)=\left|H_{e}\right|$. Thus $\mu\left(H_{e}\right)=\left|H_{e}\right|$ for all maximal subgroups $H_{e}$, and every idempotent that is not isolated is join-irreducible. If an isolated idempotent $e$ is not join-irreducible, it is the l.u.b. of two of its strict minorants. Indeed, $e$ is the l.u.b. of a set $\left\{f_{1}, \ldots, f_{k}\right\}$ of elements. Choose a maximal element $e_{i}$ in each of the sets $\left\{t \in S: f_{i} \leq t<e\right\}$. If all $e_{i}$ are equal, then $e$ cannot be the l.u.b. of $f_{i}$, and hence $e_{i} \neq e_{j}$ for some $i \neq j$. If $e_{i} \leq s$ and $e_{j} \leq s$ for some $s \in S$, then $e_{i} \leq s s^{-1} e$ and $e_{j} \leq s s^{-1} e$. If both inequalities are equalities, then $e_{i}=e_{j}$, contrary to our assumption. Thus one of these inequalities is strict, and, by the maximality of $e_{i}$ and $e_{j}$, we obtain $s s^{-1} e=e$, whence $e \leq s s^{-1}$. It follows that $e s \in H_{e}$, so that $e s=e$ and $e \leq s$. Thus $e$ is the l.u.b. of $e_{i}$ and $e_{j}$, which contradicts condition (3). Therefore, $e$ is joint-irreducible, and hence $S$ is unruly.

As we have seen in the proof of Corollary 1 , if $S$ is a semilattice of groups and $e$ is a join-irreducible idempotent of $S$, then $S_{e}$ is an intersection of the kernels of structural homomorphisms $\varphi_{e, f}$ for all $f<e$. Applying this remark to our theorem, we immediately obtain a formula for the minimal degree of a finite semilattice of groups that appeared in [2].

An inverse semigroup $S$ is called noble if it has a faithful transitive representation by one-to-one partial transformations of a set. For example, all subdirectly irreducible inverse semigroups are noble, as it is easy to prove. Minimal 
nonzero idempotents of $S$ are called primitive. It is known [8] that if $S$ is a finite noble inverse semigroup, then its subgroups $H_{e}$ and $H_{f}$ are isomorphic for any two primitive idempotents $e$ and $f$.

Corollary 2 [9]. If $S$ is a finite noble inverse subgroup, then $\delta(S)=\pi(S) \cdot \delta(G)$, where $\pi(S)$ is the number of primitive idempotents in $S$ and $G$ any maximal subgroup of $S$ which contains a primitive idempotent.

Proof. As proved in [8], a finite noble inverse semigroup $S$ has the only joinirreducible $\mathscr{D}$-class $D$. It contains all primitive idempotents of $S$, and hence $l(D)=\pi(D)$. It remains to observe that if $S$ has no zero, then all idempotents of $D$ are minimal, and hence $S_{e}=H_{e}$ for every $e \in D$. If $S$ has a zero, $S_{e}=H_{e}$. Thus $\mu(D)=\delta\left(H_{e}\right)$. It remains to apply our Theorem.

An inverse semigroup $S$ is fundamental if $(\forall e \in E)\left[\operatorname{ses}^{-1}=t e t^{-1}\right] \Rightarrow s=t$ for all $s, t \in S$.

Corollary 3 [3]. If $S$ is a fundamental inverse semigroup, then $\delta(S)$ is the number of join-irreducible idempotents in $S$. Moreover, idempotents are joinirreducible in the semilattice $E$ of idempotents of $S$ if they are join-irreducible in $S$.

Proof. Clearly, $S_{f}=\{f\}$ for every $f \in E$. Indeed, if $h \in S_{f}$, then $h e h^{-1}=$ $h f e h^{-1}=f e f^{-1}$ for every $e \in E$, for if $f e<f$ then $f e<h$, so that $h f e h^{-1}=f e h^{-1}=(h f e)^{-1}=f e=f e f^{-1}$; and if $f e=f$ then $h f e h^{-1}=$ $h f h^{-1}=f=f e f^{-1}$. Thus $h=f$. It follows that $\mu(D)=1$ for every joinirreducible $\mathscr{D}$-class $D$. Our Theorem implies that $\delta(S)=\sum\{l(D): D \in \mathbf{D}\}$. But $\sum\{l(D): D \in \mathbf{D}\}$ is merely the number of join-irreducible idempotents of $S$. To prove the last statement suppose that $e$ is join-irreducible in $S$ but not in $E$. Then $e$ is the l.u.b. of its strict minorants $f_{1}, \ldots, f_{k}$ in $E$. If $f_{i} \leq s$ for all $i$ and some $s \in S$, then $f_{i} \leq e s s^{-1}$ for all $i$, and hence $e \leq e s s^{-1}$, so that $e=e s s^{-1}$. Thus $e s \in S_{e}=\{e\}$, and hence $e s=e$ and $e \leq s$. Therefore, $e$ is the l.u.b. of $f_{1}, \ldots, f_{k}$ in $S$.

An inverse semigroup $S$ is called $E$-unitary if $e \leq s$ implies $s \in E$ for all $e \in E$ and $s \in S$. Let $K$ denote the Suschkewitsch kernel (that is, the least ideal) of $S$. Obviously, $K=H_{e}$, where $e$ is the least idempotent of $S$.

Corollary 4. If $S$ is an E-unitary inverse semigroup, then $\delta(S)=\delta(E)+\delta(K)$. Proof. Let $e$ be a join-irreducible idempotent of $S$. Consider $S_{e}$. If $e$ is the least idempotent of $S$, then $S_{e}=K, \mu\left(D_{e}\right)=\max \{1, \delta(K)\}$ and $l\left(D_{e}\right)=1$. Observe that $e$ is join-irreducible if and only if $K$ is not trivial. Otherwise, $K=\{e\}$ and $\delta(K)=0$. If $e$ is not the least idempotent, then $f<e$ for some $f \in E$, and so $f<h$ for every $h \in S_{e}$. Therefore, $h \in E$, so that $S_{e}=\{e\}$ and $\mu\left(D_{e}\right)=1$. By our Theorem, $\delta(S)=\sum l(D)+\delta(K)$, where the summation is over all join-irreducible $\mathscr{D}$-classes $D$ except $K$. Thus, $\sum l(D)$ is the number of all join-irreducible idempotents of $S$, except the least one. Every element of $E$, except the least one, is join-irreducible in $E$ if and only if it is join-irreducible in $S$. It follows that $\delta(E)=\sum l(D)$.

Corollary 5. $\delta(S \times T)=\delta(S)+\delta(T)$.

Proof. First, a few trivial remarks. Let $a$ and $b$ be the least idempotents of $S$ and $T$, respectively. Then $E_{S \times T}=E_{S} \times E_{T}$ and an idempotent is joinirreducible in $S \times T$ exactly when it is of the forms $(e, b)$ or $(a, f)$ with 
$e$ and $f$ join-irreducible in $S$ and $T$, respectively. If $(s, t) \in S \times T$, then $D_{(s, t)}=D_{s} \times D_{t}$, and hence a $\mathscr{D}$-class of $S \times T$ is join-irreducible if and only if it is of the forms $D_{s} \times H_{b}$ or $H_{a} \times D_{t}$, where $D_{s}$ and $D_{t}$ are join-irreducible $\mathscr{D}$ classes of $S$ and $T$, respectively. Also, $S_{(e, b)}=S_{e} \times\{b\}$ and $S_{(a, f)}=\{a\} \times S_{f}$ for all join-irreducible $e \in E_{S}$ and $f \in E_{T}$.

Secondly, prove that $\mu\left(D_{(e, b)}\right)=\mu\left(D_{e}\right)$ and $\mu\left(D_{(a, f)}\right)=\mu\left(D_{f}\right)$. Let $P$ be a representation of $S_{(e, b)}$ in $H_{(e, b)}$. Then $P$ is a sum of transitive representations corresponding to subgroups $F_{i}$ of $H_{(e, b)}$. Define $\operatorname{pr}_{1} F_{i}=\left\{h \in H_{e}\right.$ : $\left.(\exists t \in T)(h, t) \in F_{i}\right\}$. Let $(s, b) \in S_{(e, b)}$. Then $P(s, b)=P(e, b)$ exactly if $(x, y)^{-1}(s, b)(x, y)^{-1} \in F_{i}$ for all $(x, y) \in H_{(e, b)}=H_{e} \times K_{T}$ and all $i$. Therefore,

$$
(x, y)^{-1}(s, b)(x, y)^{-1}=\left(x^{-1} s x, y^{-1} b y\right)=\left(x^{-1} s x, b\right),
$$

and so

$$
P(s, b)=P(e, b) \Leftrightarrow(\forall i)\left(\forall x \in H_{e}\right)\left[x^{-1} s x \in \mathrm{pr}_{1} F_{i}\right] .
$$

Clearly, $\operatorname{pr}_{1} F_{i}$ is a subgroup of $H_{e}$. If we replace each $F_{i}$ by $\operatorname{pr}_{1} F_{i} \times K_{T}$, we obtain a representation $R$ of $S_{(e, b)}$ in $H_{(e, b)}$, and

$$
P(s, b)=P(e, b) \Leftrightarrow R(s, b)=R(e, b) \Leftrightarrow R_{S}(s)=R_{S}(e),
$$

where $R_{S}$ is a representation of $S_{e}$ in $H_{e}$ determined by subgroups $\mathrm{pr}_{1} F_{i}$ of $H_{e}$. Clearly, $\delta\left(R_{S}\right)=\delta(R) \leq \delta(P)$. Thus if $P$ is a minimal representation of $S_{(e, b)}$ in $H_{(e, b)}$, then $R_{S}$ is the minimal representation of $S_{e}$ in $H_{e}$, and hence $\mu\left(D_{(e, b)}\right)=\mu\left(D_{e}\right)$. The equality $\mu\left(D_{(a, f)}\right)=\mu\left(D_{f}\right)$ is proved analogously.

By our Theorem,

$$
\begin{aligned}
\delta(S \times T)= & \sum\left\{l(D) \cdot \mu(D): D \in \mathbf{D}_{S \times T}\right\} \\
= & \sum\left\{l\left(D_{(e, b)}\right) \cdot \mu\left(D_{(e, b)}\right): D_{e} \in \mathbf{D}_{S}\right\} \\
& +\sum\left\{l\left(D_{(a, f)}\right) \cdot \mu\left(D_{(a, f)}\right): D_{f} \in \mathbf{D}_{T}\right\} \\
= & \sum\left\{l\left(D_{e}\right) \cdot \mu\left(D_{e}\right): D_{e} \in \mathbf{D}_{S}\right\}+\sum\left\{l\left(D_{f}\right) \cdot \mu\left(D_{f}\right): D_{f} \in \mathbf{D}_{T}\right\} \\
= & \delta(S)+\delta(T) .
\end{aligned}
$$

Problems. (1) Study $\delta_{G}(H)$, where $H$ is a (normal) subgroup of a group $G$.

(2) Suppose that a finite inverse semigroup $S$ is a subdirect product of inverse semigroups $\left\{S_{i}: 1 \leq i \leq n\right\}$. Assume that this decomposition is irredundant (no $S_{i}$ can be omitted). Then $\delta(S) \leq \delta\left(S_{1}\right)+\delta\left(S_{2}\right)+\cdots+\delta\left(S_{n}\right)$. When is this inequality an equality? Can it be strict?

\section{REFERENCES}

1. A. H. Clifford and G. B. Preston, The algebraic theory of semigroups, Vol. II, Amer. Math. Soc., Providence, R. I., 1967.

2. David Easdown, Efficient representations of semigroups, Proc. Internat. Sympos. Theory of Regular Semigroups and Applications, University of Kerala, Trivandrum, 1987, pp. 22-46.

3. $\ldots$, The minimal faithful degree of a fundamental inverse semigroup, Bull. Austral. Math. Soc. 35 (1987), 3, 373-378.

4. J. M. Howie, An introduction to semigroup theory, Academic Press, London, 1976.

5. Mario Petrich, Inverse semigroups, Wiley, New York, 1984. 
6. Б. М. Шайн (В. М. Schein), Представления обобщённых групп (Representations of generalized groups), Известия Высших Учебных Заведении, Математика (Izv. Vyssh. Uchebn. Zaved. Matematika) 1962, no. 3, 164-176. (Russian)

7. _ـ Инверсные полугруппы, не допускающие изоморфных представлении частичными преобразованиями своих собственных подмножеств (Inverse semigroups that do not admit representations by partial transformations of their proper subsets), Теория Полугрупп и её Приложения, Издательство Саратовского Университета, Саратов (Theory of Semigroups and its Applications, vol. 3, Saratov Univ. Press, Saratov) 1974, pp. 139-148 (Russian; English transl., Amer. Math. Soc. Transl. (2) 139 (1988), 177-182).

8. B. M. Schein, Noble inverse semigroups with bisimple core, Semigroup Forum 36 (1987), 175-178.

9. __ The minimal degree of noble inverse semigroups, Contributions to General Algebra, vol. 6, Teubner, Stuttgart, 1988, pp. 247-252.

Department of Mathematical Sciences, University of Arkansas, Fayetteville, ARKANSAS 72701

E-mail address: bschein@ uafsysb.bitnet

E-mail address: bschein@ uark.edu.internet 